\title{
Material fatigue simulation using a periodic time-homogenisation method
}

\author{
Guillaume Puel* and Denis Aubry \\ Laboratoire MSSMat (Ecole Centrale Paris - CNRS UMR 8579), Grande Voie des Vignes, F-92290 \\ Châtenay-Malabry, France
}

\begin{abstract}
This paper deals with the numerical simulation of combined cycle fatigue, which is characterised by two periodic loads, whose frequencies are very different one from the other. Rather than using classical fatigue life estimations, a time transient evolution model is solved using a periodic time-homogenisation method. This latter is based on the assumption that the time scales associated with the two periodic loads are decoupled. Different results on academic as well as industrial examples are presented. An extension of the proposed method up to three time scales is eventually proposed in order to speed up the numerical simulations.
\end{abstract}

On considère ici la simulation numérique de la fatigue à cycles combinés (CCF), caractérisée par deux chargements périodiques de fréquences très différentes. Plutôt que d'utiliser les méthodes classiques de durée de vie en fatigue, on souhaite utiliser un modèle temporel d'évolution dont l'exploitation numérique est rendue possible par une méthode d'homogénéisation périodique en temps. Cette méthode repose sur une hypothèse de séparation des échelles de temps liées aux deux chargements périodiques. Différents résultats surdes exemples académiques et industriels sont présentés. Une extension de la méthode à trois échelles de temps est enfin proposée afin d'accélérer encore plus les calculs.

Keywords: periodic homogenisation; time multiscale; combined cycle fatigue; dynamics

Mots-clés: homogénéisation périodique; multiéchelles en temps; fatigue à cycles combinés; dynamique

\section{Introduction}

This paper aims at the numerical simulation of combined cycle fatigue (CCF), which is characterised by two periodic loads acting on the studied structure. Usually the frequencies associated with these two loads are very different one from the other. Since the classical fatigue life estimations do not allow to accurately take into account the chronology of the applied cycles, or the inertia effects associated with the fast cycles, it seems more relevant to use a time transient evolution model describing how internal variables such as plastic strain evolve with respect to time. Because of the significant difference between the two loading frequencies, such a numerical simulation would be too costly as is. Therefore, a periodic time-homogenisation strategy was proposed in Guennouni (1988), Guennouni and Aubry (1986) in the case of quasistatic problems. This strategy was inspired from classical schemes used in space homogenisation (Bensoussan, Lions, \& Papanicolaou, 1978; Sanchez-Palencia, 1980). Since then, similar concepts have independently emerged: 'Oscillatory Stroboscopics' (Blekhman,

*Corresponding author. Email: guillaume.puel@ecp.fr 
1994) or multiple scales homogenisation (Chen \& Fish, 2001) and have led to various applications. For example, extensions to the estimation of damage rather than plasticity have also been proposed in Devulder, Aubry, and Puel (2010), Oskay and Fish (2004). More recently, whereas the other references only dealt with quasistatic studies, the time-homogenisation strategy presented here has been extended in dynamics and validated on academic examples (Aubry \& Puel, 2010). The aim here is to apply this strategy on an actual specimen geometry, whose number of degrees of freedom is very high. The specimen proposed here comes from the European project PREMECCY dealing with CCF in aeronautics.

\section{Basis of the time-homogenisation method}

The time-homogenisation method aims at the effective numerical simulation of a structure under a high number of loading cycles, when the associated material behaviour is described by a time transient model. Even if, similarly to Guennouni (1988), Guennouni and Aubry (1986), this method is briefly presented here in the case of a generic viscoplastic behaviour, it is described in its extension to dynamics, proposed in Aubry and Puel (2010), which takes inertia effects into account.

\subsection{Time scales}

A CCF loading is assumed: typically, two periodic loads with very different frequencies are simultaneously applied to the studied structure. Two time scales are then defined:

- a slow time scale, denoted $t$, associated with the low-frequency load $F$ and

- a fast time scale, denoted $\tau$, associated with the high-frequency load $F / \xi$.

The ratio $\xi=t / \tau$ is assumed small enough so that these two time scales could be considered as independent one from the other. In this case, any time-dependent variable $\alpha$ can be written as a function of both time scales, and its time derivative leads to:

$$
\frac{\mathrm{d} \alpha}{\mathrm{d} t}=\frac{\partial \alpha}{\partial t}+\frac{1}{\xi} \frac{\partial \alpha}{\partial \tau}=\dot{\alpha}+\frac{1}{\xi} \alpha^{\prime}
$$

where $\mathrm{d} \alpha / \mathrm{d} t, \dot{\alpha}$ and $\alpha^{\prime}$ stand for the total time derivative, the partial time derivative with respect to the slow time scale $t$ and the partial time derivative with respect to the fast time scale $\tau$, respectively.

In addition, it is also assumed that any time-dependent variable $\alpha(t, \tau)$ is periodic with respect to the fast time scale $\tau$. This so-called 'quasiperiodicity' assumption is all the more justified as the ratio $\xi$ is small, and can then be written as:

$$
\alpha(t, \tau)=\alpha\left(t, \tau+\frac{\xi}{F}\right) \quad \forall t, \tau
$$

Eventually, the fast time scale averaging of variable $\alpha(t, \tau)$ is introduced in order to pass from the fast to the slow time scale:

$$
\langle\alpha\rangle=\frac{F}{\xi} \int_{0}^{\frac{\xi}{F}} \alpha(t, \tau) \mathrm{d} \tau
$$

The consequent homogenised quantity $\langle\alpha\rangle$ is a function of the slow time scale $t$ only. The residual associated with this averaging is denoted $\alpha^{*}=\alpha-\langle\alpha\rangle$, and depends on both time 
scales $t$ and $\tau$. Moreover, with the previous notations, it is possible to find an equivalent expression for the quasiperiodicity assumption (2):

$$
\left\langle\alpha^{\prime}\right\rangle=0
$$

\subsection{Asymptotic expansion of the mechanical problem}

Any field from the solution of the mechanical problem (displacement $\mathbf{u}(\mathbf{x}, t, \tau)$, stress $\sigma(\mathbf{x}, t, \tau)$, total strain $\varepsilon(\mathbf{x}, t, \tau)$ and plastic strain $\left.\varepsilon^{\mathrm{p}}(\mathbf{x}, t, \tau)\right)$ is assumed to be written in terms of an asymptotic expansion with respect to $\xi$. For example, the stress field is expressed as:

$$
\sigma(\mathbf{x}, t, \tau)=\sigma_{0}(\mathbf{x}, t, \tau)+\xi \sigma_{1}(\mathbf{x}, t, \tau)+O\left(\xi^{2}\right)
$$

The mechanical problem consists of solving, in the domain $\Omega$ associated with the studied structure, the following equations (along with null initial conditions):

$$
\begin{gathered}
\operatorname{Div}_{\mathbf{x}} \sigma+\mathbf{f}_{\mathrm{b}}=\rho \frac{\mathrm{d}^{2} \mathbf{u}}{\mathrm{d} t^{2}} \\
\sigma=\mathscr{C}\left(\varepsilon-\varepsilon^{\mathrm{p}}\right) \\
\varepsilon=\frac{1}{2}\left(\mathrm{D}_{\mathbf{x}} \mathbf{u}+\mathrm{D}_{\mathbf{x}} \mathbf{u}^{\mathrm{T}}\right) \\
\frac{\mathrm{d} \varepsilon^{\mathrm{p}}}{\mathrm{d} t}=a(\sigma)
\end{gathered}
$$

where $\rho, \mathscr{C}$ and $a$ stand for the mass density, the (linear) elasticity tensor and a generic (nonlinear) operator describing the material evolution law. $\mathbf{D i v}_{\mathbf{x}}$ and $\mathrm{D}_{\mathbf{x}}$ are the divergence and the gradient operators, respectively. $\mathbf{f}_{\mathrm{b}}(\mathbf{x}, t, \tau)$ is the body force defined in the domain $\Omega$, whereas $\mathbf{f}_{\mathbf{s}}(\mathbf{x}, t, \tau)$ is the surface force applied to its boundary $\partial \Omega$ :

$$
\sigma \mathbf{n}=\mathbf{f}_{\mathrm{s}}
$$

where $\mathbf{n}$ is the unit outer normal defined at each point of $\partial \Omega$. Without loss of generality, it can be considered that the surface force is applied only to a part of the boundary $\partial \Omega$, and that a given displacement is imposed on the complementary part.

Asymptotic expansions similar to (4) are used for each calculated field, and are introduced in Equations (5)-(9). By using the total differentiation rule (1), equations with the different powers of $\xi$ are obtained. For example, the evolution Equation (8) gives, up to order one:

$$
\frac{1}{\xi} \varepsilon_{0}^{\mathrm{p}^{\prime}}+\left(\dot{\varepsilon}_{0}^{\mathrm{p}}+\varepsilon_{1}^{\mathrm{p} \prime}\right)+\xi\left(\dot{\varepsilon}_{1}^{\mathrm{p}}+\varepsilon_{2}^{\mathrm{p} \prime}\right)+O\left(\xi^{2}\right)=a\left(\sigma_{0}\right)+\xi \mathrm{D}_{\sigma} a\left(\sigma_{0}\right) \sigma_{1}+O\left(\xi^{2}\right)
$$

with $\mathrm{D}_{\sigma} a\left(\sigma_{0}\right)$ the gradient of $a$ expressed at $\sigma_{0}$. With the assumption of decoupled scales $(\xi \ll 1)$, it can be written that, in any of these equations, the equalities have to stand for each order of $\xi$ independently from the other orders, leading to the equations detailed in Sections 2.4 and 2.5 . 


\subsection{Asymptotic expansion of the dynamic equilibrium equation}

Here is the detailed asymptotic expansion of the dynamic equilibrium equation. Using the total differentiation rule (1) allows to write the acceleration field up to order one as follows:

$$
\frac{\mathrm{d}^{2} \mathbf{u}}{\mathrm{d} t^{2}}=\frac{1}{\xi^{2}} \mathbf{u}_{0}^{\prime \prime}+\frac{1}{\xi}\left(2 \dot{\mathbf{u}}_{0}^{\prime}+\mathbf{u}_{1}^{\prime \prime}\right)+\left(\ddot{\mathbf{u}}_{0}+2 \dot{\mathbf{u}}_{1}^{\prime}+\mathbf{u}_{2}^{\prime \prime}\right)+\xi\left(\ddot{\mathbf{u}}_{1}+2 \dot{\mathbf{u}}_{2}^{\prime}+\mathbf{u}_{3}^{\prime \prime}\right)+O\left(\xi^{2}\right)
$$

Before applying this expression to Equation (5), however, the magnitude of the inertia terms should be studied first. More precisely, it can be assumed that for most usual materials, the ratio between the mass density and a norm characteristic of the magnitude of the elasticity tensor is such that:

$$
\frac{\rho}{\|\mathscr{C}\|}=\beta \xi^{2}
$$

with $\beta=o(1 / \xi)$. This assumption is correct if the inverse of the ratio $\xi$ is smaller than the pressure waves propagation celerity within the material. Such assumptions are commonly addressed in space periodic homogenisation (Sanchez-Palencia, 1980). Eventually the right side of Equation (5) reads, up to order one:

$$
\rho \frac{\mathrm{d}^{2} \mathbf{u}}{\mathrm{d} t^{2}}=\beta\|\mathscr{C}\| \mathbf{u}_{0}^{\prime \prime}+\xi \beta\|\mathscr{C}\|\left(2 \mathbf{u}_{0}^{\prime}+\mathbf{u}_{1}^{\prime \prime}\right)+O\left(\xi^{2}\right)
$$

\section{4. $1 / \xi$-order equations}

The first expression coming from the asymptotic expansions of the different equations of the mechanical problem is associated with the order $1 / \xi$ in Equation (8):

$$
\varepsilon_{0}^{\mathrm{p} \prime}=0, \text { hence } \varepsilon_{0}^{\mathrm{p}}(\mathbf{x}, t, \tau)=\varepsilon_{0}^{\mathrm{p}}(\mathbf{x}, t)
$$

This can be explained by the fact that the viscoplastic behaviour is basically (meaning at the zeroth order) a slow-evolving phenomenon when compared with the period of the fast cycles.

\subsection{Zeroth-order equations}

The next expression coming from the evolution Equation (8) is associated with order zero, where there are both the zeroth-order and first-order plastic strains. Using the quasiperiodicity relation (3) allows to make the first-order plastic strain disappear:

$$
\dot{\varepsilon}_{0}^{\mathrm{p}}=\left\langle a\left(\sigma_{0}\right)\right\rangle
$$

where we used that $\left\langle\dot{\varepsilon}_{0}^{\mathrm{p}}\right\rangle=\dot{\varepsilon}_{0}^{\mathrm{p}}$ according to Equation (12). This means that the zeroth-order plastic strain evolves as the average over a fast cycle of the evolution law expressed in terms of the zeroth-order stress field. This latter has to be considered as an 'instantaneous' quantity $\sigma_{0}(\mathbf{x}, t, \tau)$, which depends on both time scales.

In order to evaluate this quantity, the first step consists in defining the zeroth-order homogenised equations coming from Equations (5)-(7), (9) by using the same technique as for the evolution Equation (8): 


$$
\begin{gathered}
\operatorname{Div}_{\mathbf{x}}\left\langle\sigma_{0}\right\rangle+\left\langle\mathbf{f}_{\mathrm{b}}\right\rangle=\mathbf{0} \\
\left\langle\sigma_{0}\right\rangle=\mathscr{C}\left(\left\langle\varepsilon_{0}\right\rangle-\varepsilon_{0}^{\mathrm{p}}\right) \\
\left\langle\varepsilon_{0}\right\rangle=\frac{1}{2}\left(\mathrm{D}_{\mathbf{x}}\left\langle\mathbf{u}_{0}\right\rangle+\mathrm{D}_{\mathbf{x}}\left\langle\mathbf{u}_{0}\right\rangle^{\mathrm{T}}\right) \\
\left\langle\sigma_{0}\right\rangle \mathbf{n}=\left\langle\mathbf{f}_{\mathrm{s}}\right\rangle
\end{gathered}
$$

This shows that the zeroth-order homogenised equilibrium equation is a quasistatic equation, where the inertia terms are not present any more.

The second step consists of solving the residual equations coming from the previous homogenisation process:

$$
\begin{gathered}
\operatorname{Div}_{\mathbf{x}} \sigma_{0}^{*}+\mathbf{f}_{\mathrm{b}}^{*}=\beta\|\mathscr{C}\| \mathbf{u}_{0}^{* \prime \prime} \\
\sigma_{0}^{*}=\mathscr{C} \varepsilon_{0}^{*} \\
\varepsilon_{0}^{*}=\frac{1}{2}\left(\mathrm{D}_{\mathbf{x}} \mathbf{u}_{0}^{*}+\mathrm{D}_{\mathbf{x}} \mathbf{u}_{0}^{* \mathrm{~T}}\right) \\
\sigma_{0}^{*} \mathbf{n}=\mathbf{f}_{\mathrm{s}}^{*}
\end{gathered}
$$

where Equations (14)-(17) have been subtracted from the zeroth-order equations corresponding to the initial ones (5)-(7), (9). The previous equations correspond to the definition of a dynamic linear elastic problem, whose solution $\sigma_{0}^{*}(\mathbf{x}, t, \tau)$ is completely decoupled from the solution of Equations (14)-(17) and can be computed once for all. As a result, Equations (14)-(17) are solved along with Equation (8) using the fact that $\sigma_{0}(\mathbf{x}, t, \tau)=\left\langle\sigma_{0}\right\rangle(\mathbf{x}, t)+\sigma_{0}^{*}(\mathbf{x}, t, \tau)$.

\section{Application to a simple example}

In order to show the validity as well as the efficiency of the method, the following academic example is proposed: a cylindrical bar of length $L$ withstands a normal loading defined as the sum of a slow-evolving load of frequency $F$ and a fast-evolving load of frequency $F / \xi$. The chosen material law is viscoplastic with two hardenings as defined in Lemaitre and Chaboche (1990).

\subsection{Reference problem}

The reference problem consists of the transient equations, which have not yet been homogenised. The dynamic equilibrium equation is scalar and deals with the longitudinal displacement $u(x, t, \tau)$ of the bar's median axis as well as with the normal stress $\sigma(x, t, \tau)$ within the bar:

$$
\frac{\partial \sigma}{\partial x}=\rho \frac{\mathrm{d}^{2} u}{\mathrm{~d} t^{2}}
$$

where $x \in(0, L)$. Here, it is assumed that there is no body force within the bar, and that the only loading is the surface force $f_{\mathrm{s}}(t, \tau)$ applied in $x=L$. In $x=0$, the bar is clamped. The elastic constitutive relation is given by:

$$
\sigma=E\left(\frac{\partial u}{\partial x}-\varepsilon^{\mathrm{p}}\right)
$$


where $E$ and $\varepsilon^{\mathrm{p}}(x, t, \tau)$ are the Young's modulus and the longitudinal plastic strain, respectively. The evolution law is viscoplastic with two non-linear hardenings, which in its uniaxial expression, coming from Lemaitre and Chaboche (1990), consists of the following equations (along with null initial conditions):

$$
\begin{gathered}
\frac{\mathrm{d} p}{\mathrm{~d} t}=\left\langle\frac{|\sigma-X|-R-k}{K}\right\rangle_{+}^{n} \\
\frac{\mathrm{d} \varepsilon^{\mathrm{p}}}{\mathrm{d} t}=\frac{\mathrm{d} p}{\mathrm{~d} t} \operatorname{sign}(\sigma-X) \\
\frac{\mathrm{d} X}{\mathrm{~d} t}=\frac{2}{3} C \frac{\mathrm{d} \varepsilon^{\mathrm{p}}}{\mathrm{d} t}-\gamma_{0} \frac{\mathrm{d} p}{\mathrm{~d} t} X \\
\frac{\mathrm{d} R}{\mathrm{~d} t}=b(Q-R) \frac{\mathrm{d} p}{\mathrm{~d} t}
\end{gathered}
$$

where $\langle\alpha\rangle_{+}=(1+\operatorname{sign} \alpha) \alpha / 2$ is the positive part of $\alpha(t, \tau) . \mathrm{d} p / \mathrm{d} t(x, t, \tau), X(x, t, \tau)$ and $R(x, t, \tau)$ stand for the equivalent plastic strain rate, the (uniaxial) kinematic hardening variable and the (uniaxial) isotropic hardening variable, respectively. $k, K, n, C, \gamma_{0}, b$ and $Q$ are material parameters, which are constant at given temperature.

\subsection{Zeroth-order homogenised equations}

When using the same technique as in Section 2.4, the same conclusion is obtained, that is zeroth-order viscoplasticity is a slow-evolving phenomenon:

$$
\begin{array}{cl}
p_{0}(x, t, \tau)=p_{0}(x, t) & \varepsilon_{0}^{\mathrm{p}}(x, t, \tau)=\varepsilon_{0}^{\mathrm{p}}(x, t) \\
X_{0}(x, t, \tau)=X_{0}(x, t) & R_{0}(x, t, \tau)=R_{0}(x, t)
\end{array}
$$

Then by applying the steps of Section 2.5, the zeroth-order homogenised and residual equilibrium equations are the following ones:

$$
\begin{gathered}
\frac{\partial}{\partial x}\left[E\left(\frac{\partial\left\langle u_{0}\right\rangle}{\partial x}-\varepsilon_{0}^{\mathrm{p}}\right)\right]=0 \\
\frac{\partial}{\partial x}\left[E \frac{\partial u_{0}^{*}}{\partial x}\right]=\beta E u_{0}^{* \prime \prime}
\end{gathered}
$$

along with the following boundary conditions in $x=0$ and $L$, respectively:

$$
\left\langle u_{0}\right\rangle=0 \quad \text { and } \quad E\left(\frac{\partial\left\langle u_{0}\right\rangle}{\partial x}-\varepsilon_{0}^{\mathrm{p}}\right)=\left\langle f_{\mathrm{s}}\right\rangle
$$




$$
u_{0}^{*}=0 \quad \text { and } \quad E \frac{\partial u_{0}^{*}}{\partial x}=f_{\mathrm{s}}^{*}
$$

Eventually the zeroth-order homogenised evolution laws are given by:

$$
\begin{gathered}
\dot{p}_{0}=\left\langle\left\langle\frac{\left|\sigma_{0}-X_{0}\right|-R_{0}-k}{K}\right\rangle_{+}^{n}\right\rangle \\
\dot{\varepsilon}_{0}^{\mathrm{p}}=\left\langle\left\langle\frac{\left|\sigma_{0}-X_{0}\right|-R_{0}-k}{K}\right\rangle_{+}^{n} \operatorname{sign}\left(\sigma_{0}-X_{0}\right)\right\rangle \\
\dot{X}_{0}=\frac{2}{3} C \dot{\varepsilon}_{0}^{\mathrm{p}}-\gamma_{0} \dot{p}_{0} X_{0} \\
\dot{R}_{0}=b\left(Q-R_{0}\right) \dot{p}_{0}
\end{gathered}
$$

where the instantaneous zeroth-order stress can be expressed as:

$$
\sigma_{0}(x, t, \tau)=E\left(\frac{\partial\left\langle u_{0}\right\rangle}{\partial x}(x, t)+\frac{\partial u_{0}^{*}}{\partial x}(x, t, \tau)-\varepsilon_{0}^{\mathrm{p}}(x, t)\right)
$$

\subsection{Numerical results}

Equations (24)-(31) are solved using a time-dependent finite element model with respect to the slow time variable $t$. It is assumed that the force applied in $x=L$ can be written as:

$$
\left\langle f_{\mathrm{s}}\right\rangle(t)=f_{0}+f_{1} \cos 2 \pi F t \quad \text { and } \quad f_{\mathrm{s}}^{*}(\tau)=f_{2} \cos 2 \pi F \tau
$$

where $f_{0}, f_{1}$ and $f_{2}$ are given constants, and the slow and fast frequencies are $F=0.05 \mathrm{~Hz}$ and $F / \xi=500 \mathrm{~Hz}$, respectively. This example, which is consistent with what is applied in CCF, allows to write that $u_{0}^{*}$ is a function of $x$ and $\tau$ only. Figure 1 depicts the surface force that is applied on the numerical examples below: the high discrepancy between the two frequencies only allows to see the envelope of the surface load.

The elastic dynamic Equation (25) along with Equation (27) can then be solved independently from the other equations, that is, it can be solved once for all, in a preprocessing step, over the fast period. This fast time solution $u_{0}^{*}$ is then used to estimate the instantaneous stress (32) required in Equations (28)-(31). The fast time scale averaging of $\alpha(t, \tau)$ is then computed using a quadrature rule, such as the classical trapezoidal rule:

$$
\langle\alpha\rangle \approx \frac{1}{N}\left(\frac{\alpha(t, 0)}{2}+\sum_{j=1}^{N-1} \alpha\left(t, \frac{j}{N} \frac{\xi}{F}\right)+\frac{\alpha\left(t, \frac{\xi}{F}\right)}{2}\right)
$$

with $N$ chosen as a trade-off between the accuracy of the estimate and the calculation cost. Eventually, the remaining Equations (24), (26), (28)-(31) depend only on the slow time variable $t$. 


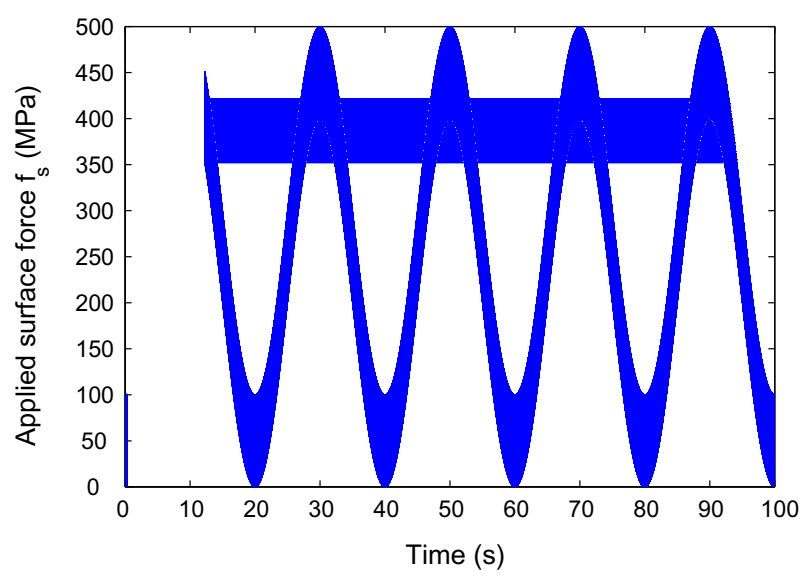

Figure 1. Applied surface force, with $f_{0}=250 \mathrm{MPa}, f_{1}=-200 \mathrm{MPa}$ and $f_{2}=-50 \mathrm{MPa}$.

The simulations are carried out for a bar made of TA6V titanium alloy, whose material properties at $350^{\circ} \mathrm{C}$ are given in Lemaitre and Chaboche (1990). In order to validate the proposed time for homogenisation method, a reference calculation, which consists in directly solving the equations from Section 3.1, is carried out on the time interval $[0,20] \mathrm{s}$ corresponding to the first slow load period. The accuracy of this calculation is guaranteed by choosing a very fine time step $\left(10^{-4} \mathrm{~s}\right)$ corresponding to a 20th of the fast load period. The associated results will then be compared with these obtained with a zeroth-order time-homogenised calculation on the same time interval, but using a 0.04 s-time step, hence reducing the number of iterations by 400 .

The results of these two simulations are depicted in Figure 2. The zeroth-order timehomogenised plastic strain (blue dotted line) is in very good agreement with the reference plastic strain in $x=0$ (red dashed line); this is confirmed by Table 1 . In order to evaluate the effect of taking the inertia terms into account in the equilibrium Equation (5), additional

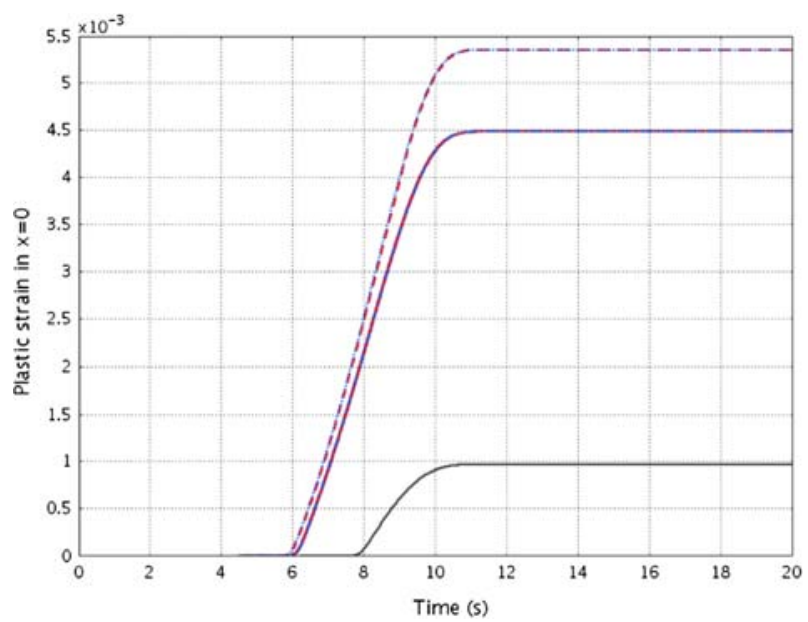

Figure 2. Plastic strain in $x=0$ : reference solutions (in red) and time-homogenised (in blue) solutions (dynamic and quasistatic) and quasistatic solution when the fast cycles are not taken into account in the homogenization process (in black). 
Table 1. Comparison of the different calculations for the plastic strain at $t=20 \mathrm{~s}$.

\begin{tabular}{lcc}
\hline Simulations & $x=0$ & $x=L$ \\
\hline Reference dynamic & $5.361 \cdot 10^{-3}$ & $4.654 \cdot 10^{-3}$ \\
Zeroth-order time-homogenized dynamic & $5.389 \cdot 10^{-3}$ & $4.595 \cdot 10^{-3}$ \\
Reference quasistatic & $4.491 \cdot 10^{-3}$ & $4.491 \cdot 10^{-3}$ \\
Zeroth-order time-homogenized quasistatic & $4.493 \cdot 10^{-3}$ & $4.493 \cdot 10^{-3}$ \\
\hline
\end{tabular}

calculations have been carried out in the quasistatic case, that is by assuming that $\rho=0$ in Equation (22) or that $\beta=0$ in Equation (25). Figure 2 and Table 1 show the significance of the inertia terms at the studied frequencies as well as the good match between the results of the reference calculation (red continuous line) and of the time-homogenised calculation (blue dashed-dotted line). Eventually, the necessity of correctly taking the fast cycles into account in the time-homogenisation process is demonstrated by the black curve, where the plastic strain has been calculated by assuming that $\varepsilon_{0}^{\mathrm{p}}=a\left(\left\langle\sigma_{0}\right\rangle\right)$ instead of Equation (13), that is without taking into account the fast cycles in the plastic strain evaluation. Figure 3, which is a zoom of Figure 2, makes easier the comparison between the reference calculation (in red) and the zeroth-order time-homogenised calculation (in blue): whereas the reference plastic strain increases step by step (each step corresponding to each fast cycle), the time-homogenised plastic strain evolves more smoothly and remains close to the reference one.

These results show the efficiency of the time-homogenisation method allowing to reduce by 400 the number of calculated time steps. It is then possible to solve problems, which would be impossible to calculate as is. Figure 4 shows how the zeroth-order time-homogenised plastic strain evolves for a time interval of one hour, which is equivalent to applying 180 slow cycles along with 1,800,000 fast cycles. With the time-homogenisation method, only 90,000 time steps of length $0.04 \mathrm{~s}$ are calculated, instead of the 36,000,000 time steps required for a reference calculation using a time step of $10^{-4} \mathrm{~s}$.

It is important to note that there is no limitation regarding the fast load amplitude comparatively to the slow load amplitude; the previous results have been calculated when the ratio of the former over the latter was one-fourth. It is even possible to study the limit case when there is no slow load applied to the structure, that is the high cycle fatigue (HCF) case. Once again, the agreement between reference and time-homogenised calculations is very good, as shown by Figure 5 in the quasistatic case for loading frequencies of 0.001 and $10 \mathrm{~Hz}$.

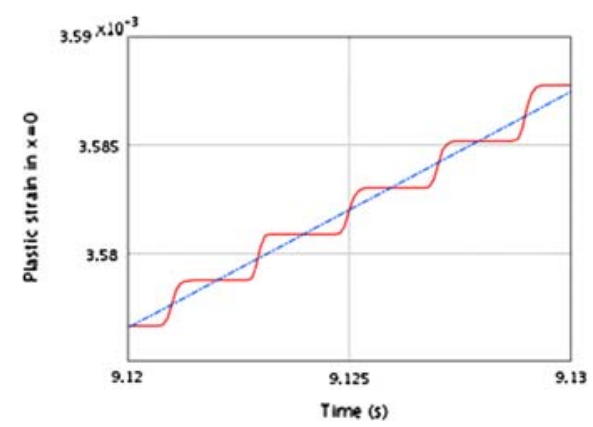

Figure 3. Plastic strain in $x=0$ (zoom). 


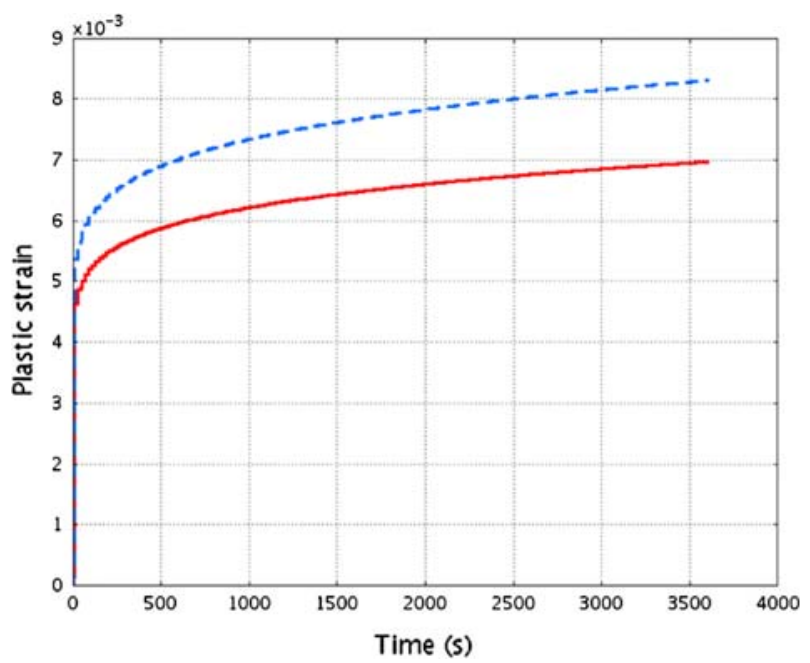

Figure 4. Zeroth-order time-homogenised plastic strain in $x=0$ (blue-dashed line) and in $x=L$ (red continuous line).

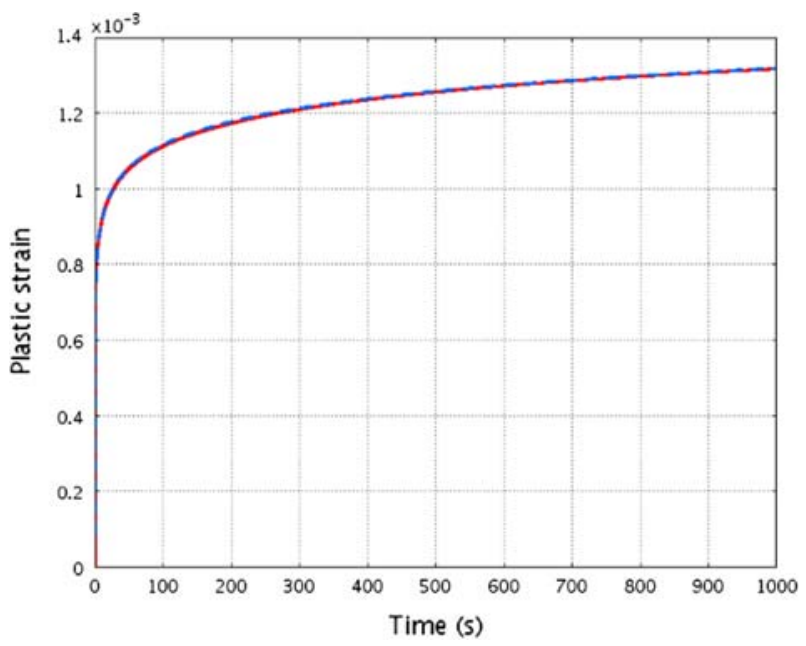

Figure 5. Plastic strain in HCF: reference calculation (red continuous line) and time-homogenised calculation (blue-dashed line).

Ultimately, it is possible to evaluate a fatigue criterion based on the plastic strain evolution; if it is assumed that fracture occurs beyond a given threshold $\varepsilon_{m}^{\mathrm{p}}$, the cycles limit number is obtained by calculating the time $t_{m}$ such that:

$$
\int_{0}^{t_{m}}\left\langle\left\langle\frac{\left|\sigma_{0}-X_{0}\right|-R_{0}-k}{K}\right\rangle_{+}^{n} \operatorname{sign}\left(\sigma_{0}-X_{0}\right)\right\rangle \mathrm{d} t=\varepsilon_{m}^{\mathrm{p}}
$$




\section{Application to an industrial example}

The time-homogenisation method is applied to an actual geometry, used in the European project PREMECCY, and depicted in Figure 6.

The specimen, made of titanium alloy, is used to characterise CCF in turbine fans, and is loaded at one of its ends, whereas the other end is clamped. Equations (5)-(7) and (9) are considered, as well as the viscoplasticity model with two hardenings (Lemaitre \& Chaboche, 1990) in its tridimensional expression. Two different calculations are carried out in order to demonstrate the effect of the inertia terms in the plastic strain evolution. In both cases, a CCF loading is applied with a low-frequency normal load along with a high-frequency transverse load at the same end. For the first calculation, the high frequency $(1,441 \mathrm{~Hz})$ corresponds to the first bending mode of the specimen, whereas the low frequency is chosen at $0.1441 \mathrm{~Hz}$. Because the specimen is excited at one of its resonance frequencies, Rayleigh damping $\left(10^{-3}\right)$ is added to the dynamic Equation (5). For the second calculation, the high frequency is chosen at $10 \mathrm{~Hz}$, which is much lower than the first natural frequency of the specimen, whereas the low frequency is $0.001 \mathrm{~Hz}$. In both cases, 100 slow cycles and 1,000,000 fast cycles are calculated. It is then seen that the average longitudinal plastic strain within the specimen is much higher for the first calculation $\left(1.25 \cdot 10^{-6}\right)$ than for the second one $\left(2.90 \cdot 10^{-7}\right)$. This result highlights how much the resonant excitation impacts the irreversible behaviour of the specimen, and eventually the fatigue life. However, the computation times for these two calculations remain still high (about one day on a typical workstation), which means that, if more slow cycles are to be computed, the CCF life estimation would still remain difficult to be evaluated.

\section{Towards a three time scale homogenisation method}

Regarding the previous remark, one way to further improve the computational cost would be to use the fact that the low-frequency load is periodic. Then, if it is considered that the zeroth-order time-homogenised problem (13)-(17) is the new reference problem, it is possible to apply once again the time-homogenisation strategy to this new reference problem. This is equivalent to say that we consider a third time scale $\theta$ such that $t=\theta / \eta$, where $\eta$ can be arbitrarily small. When considering for $\alpha(\theta, t)$ that:

$$
\frac{\mathrm{d} \alpha}{\mathrm{d} \theta}=\frac{\partial \alpha}{\partial \theta}+\frac{1}{\eta} \frac{\partial \alpha}{\partial t}=\breve{\alpha}+\frac{1}{\eta} \dot{\alpha} \quad \text { and } \quad[\dot{\alpha}]=F \int_{0}^{\frac{1}{F}} \dot{\alpha}(\theta, t) \mathrm{d} t=0
$$

and using an asymptotic expansion of each zeroth-order time-homogenised variable, such as the zeroth-order time-homogenised stress field:

$$
\left\langle\sigma_{0}\right\rangle(\mathbf{x}, \theta, t)=\sigma_{00}(\mathbf{x}, \theta, t)+\eta \sigma_{01}(\mathbf{x}, \theta, t)+O\left(\eta^{2}\right)
$$

it can be shown that:

$$
\begin{gathered}
\varepsilon_{00}^{\mathrm{p}}(\mathbf{x}, \theta, t)=\varepsilon_{00}^{\mathrm{p}}(\mathbf{x}, \theta) \\
\breve{\varepsilon}_{00}^{\mathrm{p}}=\left[\left\langle a\left(\sigma_{00}+\sigma_{0}^{*}\right)\right\rangle\right]
\end{gathered}
$$




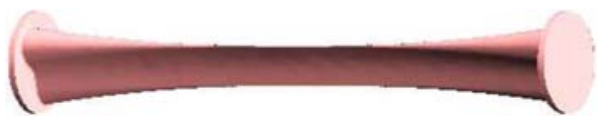

Figure 6. Specimen used in the European project PREMECCY.

$$
\begin{gathered}
\operatorname{Div}_{\mathbf{x}}\left[\sigma_{00}\right]+\left[\left\langle\mathbf{f}_{\mathrm{b}}\right\rangle\right]=\mathbf{0} \\
{\left[\sigma_{00}\right]=\mathscr{C}\left(\left[\varepsilon_{00}\right]-\varepsilon_{00}^{\mathrm{p}}\right)} \\
{\left[\varepsilon_{00}\right]=\frac{1}{2}\left(\mathrm{D}_{\mathbf{x}}\left[\mathbf{u}_{00}\right]+\mathrm{D}_{\mathbf{x}}\left[\mathbf{u}_{00}\right]^{\mathrm{T}}\right)} \\
{\left[\sigma_{00}\right] \mathbf{n}=\left[\left\langle\mathbf{f}_{\mathrm{s}}\right\rangle\right]}
\end{gathered}
$$

and that a residual problem, analogous to Equations (18)-(21) in the quasistatic case, can be defined for $\tilde{\sigma}_{00}=\sigma_{00}-\left[\sigma_{00}\right]$ and associated fields $\tilde{\varepsilon}_{00}$ and $\tilde{\mathbf{u}}_{00}$.

An example consisting of a one-day simulation of the same bar as in Section 3.3 is proposed in the quasistatic case, with a $10 \mathrm{~Hz}$ fast frequency, whereas the slow frequency is $0.001 \mathrm{~Hz}$. Figure 7 shows for the plastic strain evolution, the comparison between the initial two time scale and the newly proposed three time scale strategies. The agreement is quite satisfactory, provided that the time steps are judiciously chosen in the three time scale method: in this example, the solution is computed every second until the first slow loading cycle is over (at $t=200 \mathrm{~s}$ ), then every 200th second. It seems indeed to be mandatory that the time steps are small at the beginning of the calculation in order to ensure a good convergence. Concerning the computation cost, the three time scale method only needs about 500 time

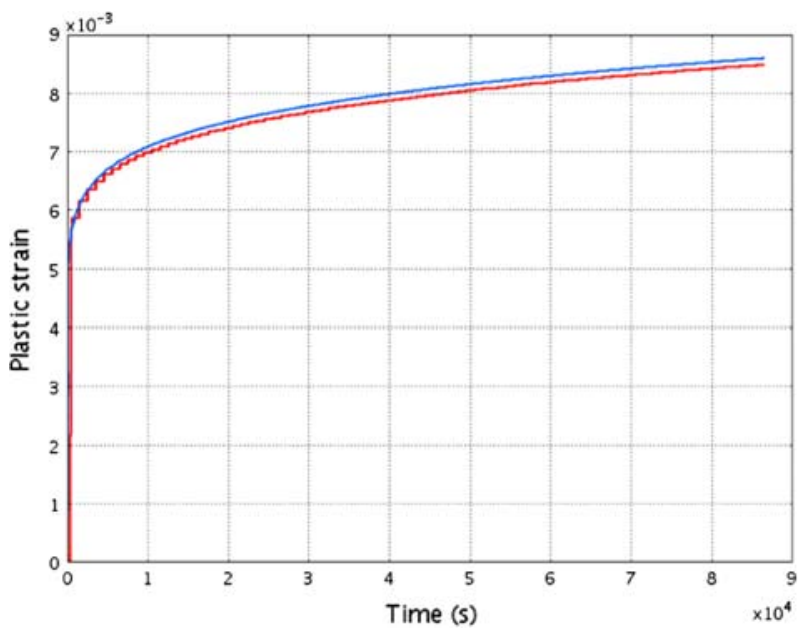

Figure 7. Plastic strain: two time scale (red line) and three time scale (blue line) time-homogenised solutions. 
steps, whereas the two time scale method requires around 50,000 time steps, which means that a huge cost reduction is obtained.

\section{Conclusion}

The efficiency of the periodic time-homogenisation method has been demonstrated in the case of academic as well as industrial examples. It allows to solve the different equations at the slow time scale only, by taking into account the averaged effect of the fast frequency cycles in the homogenised solution. In order to evaluate fatigue life, which is associated with a very high number of cycles, a three-time step strategy is proposed to speed up the calculations. Work is in progress to further improve the associated predictions as well as to validate it in the dynamic case.

\section{Note}

1. PREdictive MEthods for Combined CYcle fatigue in gas turbines, EU Project (6th RTD Framework programme), http://premeccy.mecc.polimi.it/.

\section{References}

Aubry, D., \& Puel, G. (2010). Two-timescale homogenization method for the modeling of material fatigue. IOP Conference Series: Material Science and Engineering, 10, 012113.

Bensoussan, A., Lions, J.L., \& Papanicolaou, G. (1978). Asymptotic Analysis for Periodic Structures. Burlington, MA: Elsevier.

Blekhman, I.I. (1994). Vibrational mechanics. Physmatlit. Moscow (In Russian, English edition by World Scientific in 2000).

Chen, W., \& Fish, J.A. (2001). Dispersive model for wave propagation in periodic heterogeneous media based on homogenization with multiple spatial and temporal scales. Journal of Applied Mechanics, 68(2), 153-161.

Devulder, A., Aubry, D., \& Puel, G. (2010). Two-time scale fatigue modelling: Application to damage. Computational Mechanics, 45(6), 637-646.

Guennouni, T. (1988). Sur une méthode de calcul de structures soumises à des chargements cycliques: l'homogénéisation en temps (On a method for calculating structures under cyclic loadings: time homogenisation). Mathematical Modelling and Numerical Analysis, 22(3), 417-455.

Guennouni T., \& Aubry, D. (1986). Réponse homogénéisée en temps de structures sous chargements cycliques, Comptes rendus de l'Académie des sciences (Equivalent response of structures under cyclic loadings). Série II. Mécanique, physique, chimie, sciences de l'univers, sciences de la terre, 303 (20): $1765-1768$.

Lemaitre, J., \& Chaboche, J.-L. (1990). Mechanics of solid materials. Cambridge: Cambridge University Press.

Oskay, C., \& Fish, J. (2004). Fatigue life prediction using 2-scale temporal asymptotic homogenization. International Journal for Numerical Methods in Engineering, 61(3), 329-359.

Sanchez-Palencia E. (1980). Non-homogeneous media and vibration theory. Berlin: Springer. 